\title{
Cisplatin alters nitric oxide synthase levels in human ovarian cancer cells: involvement in p53 regulation and cisplatin resistance
}

\author{
EL Leung ${ }^{1,2,3}$, M Fraser ${ }^{1,2,4}$, RR Fiscus ${ }^{3,5}$ and BK Tsang ${ }^{*, 1,2}$ \\ 'Reproductive Biology Unit and Division of Gynecologic Oncology, Department of Obstetrics and Gynecology and Cellular and Molecular Medicine, \\ University of Ottawa, Ottawa, Ontario, Canada KIY 4E9; ${ }^{2}$ Chronic Disease Program, Ottawa Health Research Institute, Ottawa, Ontario, Canada KIY \\ 4E9; ${ }^{3}$ Department of Physiology, Faculty of Medicine and Centre of Gerontology and Geriatrics, Chinese University of Hong Kong, Shatin, NT, Hong Kong
}

\begin{abstract}
The present study determines if ( 1 ) basal protein levels of nitric oxide (NO) synthases (eNOS, iNOS, and nNOS) are different in cisplatin-sensitive (OV2008) and counterpart cisplatin-resistant (CI 3*) human ovarian cancer cells, (2) cisplatin alters NOS levels, (3) $\mathrm{NO}$ donor causes apoptosis and p53 upregulation, (4) NO donor sensitises Cl $3^{*}$ cells to cisplatin via p53 upregulation (determined by p53 siRNA gene-knockdown), and (5) inhibition of endogenous NOS alters cisplatin-induced apoptosis. Basal iNOS levels were higher in OV2008 cells than in CI3* cells. Cisplatin upregulated iNOS, but dramatically reduced eNOS and nNOS, in OV2008 cells only. Failure of cisplatin to upregulate iNOS and downregulate eNOS/nNOS in cisplatin-resistant CI $3 *$ cells may be an aetiological factor in the development of resistance. The NO donor S-nitroso-N-acetylpenicillamine (SNAP) increased p53 protein levels and induced apoptosis in both cell types, and enhanced cisplatin-induced apoptosis in CI3* cells in a p53-dependent manner (i.e., enhancement blocked by p53 siRNA). Specific iNOS inhibitor I400W partially blocked cisplatin-induced apoptosis in OV2008 cells. In cisplatin-resistant $\mathrm{Cl} 3^{*}$ cells, blocking all NOSs with $N^{\mathrm{G}}$-amino-L-arginine dramatically changed these cells from cisplatin-resistant to cisplatin-sensitive, greatly potentiating cisplatin-induced apoptosis. The data suggest important roles for the three NOSs in regulating chemoresistance to cisplatin in ovarian cancer cells.

British Journal of Cancer (2008) 98, 1803-1809. doi: I0.1038/sj.bjc.6604375 www.bjcancer.com
\end{abstract}

Published online 27 May 2008

(c) 2008 Cancer Research UK

Keywords: nitric oxide; ovarian cancer; apoptosis; NOS; p53; cisplatin

Cisplatin (CDDP) is a widely used first-line chemotherapeutic agent for ovarian cancer (Siddik, 2003), and the induction of apoptosis is a key determinant of CDDP sensitivity. Indeed, we and others have demonstrated that failure of CDDP to induce apoptosis is a major component of CDDP resistance (Sasaki et al, 2000; Yuan et al, 2003; Fraser et al, 2003a). CDDP induces DNA crosslinks, upregulation of $\mathrm{p} 53$, and subsequent $\mathrm{p} 53$-dependent apoptosis (Siddik, 2003). However, in CDDP-resistant ovarian cancer cells, failure to properly regulate p53 can contribute to the resistance (Fraser et al, 2003a,b). Furthermore, basal activity of soluble guanylyl cyclase (sGC), an intracellular receptor for nitric oxide (NO), suppresses apoptosis in ovarian cancer cells, partly via inhibition of p53 accumulation and activation (Fraser et al, 2006). We hypothesised that basal sGC activity and regulation of p53 may depend on endogenous NO formed by one of the three NO synthases (NOSs), eNOS (also called NOS3), iNOS (also called NOS2), or nNOS (also called NOS1) and that resistance to CDDP in ovarian cancer cells may involve altered expression of the NOSs.

*Correspondence: Dr BK Tsang, Chronic Disease Program, Ottawa Health Research Institute, 725 Parkdale Avenue, Ottawa, Ontario, Canada KIY 4E9;

E-mail: btsang@ohri.ca

${ }^{4}$ Current address: Ontario Cancer Institute, Princess Margaret Hospital, Toronto, Ontario, Canada M5G 2M9.

${ }^{5}$ Current address: Nevada Cancer Institute, One Breakthrough Way, Las Vegas, NV, 89/35, USA.

Received 3 January 2008; revised 7 April 2007; accepted 8 April 2008; published online 27 May 2008
At high concentrations (typically $>100 \mu \mathrm{M}$ ), NO donors generate toxic concentrations of NO (e.g., >100 nM), inducing apoptosis in many mammalian cells (Fiscus, 2002; Fiscus et al, 2002), including cancer cells (Wink et al, 1998). However, NO at lower, more physiological levels (e.g., 1-20 nM) often has the opposite effect, preventing (or attenuating) the onset of apoptosis in many mammalian cells, including lung fibroblasts (Wink et al, 1993), human B lymphocytes (Mannick et al, 1994), and many normal and transformed neural cells (Fiscus, 2002; Fiscus et al, 2002). In most cells, the anti-apoptotic effects of low-level NO are attributed to the stimulation of sGC with subsequent elevation of cyclic guanosine monophosphate (cGMP) levels and increased activation of protein kinase $\mathrm{G}$ (PKG). The anti-apoptotic role of cGMP/PKG signalling pathway has been further established using natriuretic peptides, atrial natriuretic peptide, and B-type/brain natriuretic peptide (BNP), which activate cGMP/PKG via an alternate pathway (i.e., activation of particulate guanylyl cyclase). Atrial natriuretic peptide and BNP are exceptionally effective inhibitors of stress-induced apoptosis in PC-12 phaeochromocytoma and in NG108-15 neuroblastoma-glioma hybrid cells, including suppressing the toxic/pro-apoptotic effects of high-level NO (Fiscus et al, 2001; Cheng Chew et al, 2003). Even basal levels of cGMP, generated primarily by basal sGC activity, and subsequent partial activation of PKG are sufficient (in fact, necessary) to prevent spontaneous apoptosis in certain cells, including NG108-15 cells (Fiscus, 2002; Fiscus et al, 2002), immortalised uterine epithelial cells (Chan and Fiscus, 2003), and human ovarian cancer cells (Fraser et al, 2006). Because sGC is activated by $\mathrm{NO}$ at very low concentrations (i.e., $50 \%$ of maximal 
sGC activity stimulated by only $4 \mathrm{~nm}$ NO; Bellamy et al, 2002), low endogenous level of NO, generated by one of the three NOSs, may be responsible for stimulating basal sGC activity and inhibiting apoptosis in these cells.

Previously, melanoma cells (Tang and Grimm, 2004), transformed $\beta$-cell line RINm5F (Tejedo et al, 2001), and transformed human leukocytes (Mannick et al, 1997) were reported to generate endogenous NO capable of suppressing apoptosis. Endogenous NO is also believed to be responsible for aggressive tumour progression, metastasis, and poor survival in cancer patients with tumours exhibiting increased iNOS expression (Thomsen and Miles, 1998). However, other studies focusing on ovarian cancer have shown that overexpression of iNOS correlates with increased apoptosis (Son and Hall, 2000; Rieder et al, 2001). Thus, endogenous NO may contribute to either anti-apoptotic or pro-apoptotic effects in cancer cells, most likely depending on cell type, local NO levels, source of NO production, and other conditions (e.g., concurrent overproduction of superoxide anion, which can combine with NO to form the toxic prooxidant peroxynitrite) (Fiscus, 2002; Fiscus et al, 2002).

The present study determines whether eNOS, iNOS, and nNOS are involved in regulating p53 accumulation and CDDP resistance in ovarian cancer cells. CDDP-sensitive OV2008 cells and its resistant variant $\mathrm{C}_{13}{ }^{*}$ cells were used. Effects of NO donor and NOS inhibitors on CDDP-induced p53 regulation and apoptosis were also studied. Overall, the present study shows that all three isoforms of NOS are expressed in both cell lines, but protein levels are differentially regulated by CDDP in CDDP-sensitive and CDDP-resistant cells. Furthermore, the data suggest that iNOS contributes to CDDP-induced apoptosis in CDDP-sensitive cells, whereas eNOS/nNOS contributes (in a p53-independent manner) to chemoresistance by suppressing CDDP-induced apoptosis in isogenic CDDP-resistant cells.

\section{MATERIALS AND METHODS}

\section{Reagents}

Chemicals were purchased from the following suppliers: Hoechst 33258, phenylmethlysulphonyl fluoride (PMSF), sodium orthovanadate $\left(\mathrm{Na}_{3} \mathrm{VO}_{4}\right)$, and aprotinin (Sigma, St Louis, MO, USA); $S$-nitroso$\mathrm{N}$-acetylpenicillamine (SNAP) and rabbit monoclonal anti-iNOS, anti-eNOS, and anti-nNOS antibodies (Calbiochem-Novabiochem, La Jolla, CA, USA); mouse monoclonal anti-p53, clone DO-1 (Santa Cruz Biotechnologies, Santa Cruz, CA, USA); mouse monoclonal anti-GAPDH antibody (ab8245) (Abcam, Cambridge, UK); p53siRNA (Cell Signaling Technology, Beverly, MA, USA); pre-stained SDS-PAGE standards (Bio-Rad, Hercules, CA, USA); scrambled control siRNA (Dharmacon, Lafayette, CO, USA); specific iNOS inhibitor $1400 \mathrm{~W}$ and general NOS inhibitor $N^{\mathrm{G}}$-amino-L-arginine (L-NAA) (Alexis Biochemicals, San Diego, CA, USA).

\section{Cell lines and culture}

Two counterpart human epithelial ovarian cancer cell lines, OV2008 and $\mathrm{C} 3^{*}$ cells, were used. Cisplatin-sensitive OV2008 cells was derived from ovarian cystadenocarcinoma patient without prior chemotherapy, and its CDDP-resistant variant C13* was established after in vitro CDDP challenge. Wild-type TP53 status of these cells has been previously demonstrated by direct sequencing (Fraser et al, 2008). Cells were cultured as described previously (Fraser et al, 2006).

\section{Hoechst 33258 staining}

Cells were handled and stained with Hoechst 33258 as previously described (Fraser et al, 2006). Counting was carried out blinded to sample identity, to reduce experimental bias.

\section{Protein extraction and western blotting}

Cells were pelleted and lysed in ice-cold lysis buffer $(\mathrm{pH} 7.4)$ containing $50 \mathrm{~mm}$ Hepes, $150 \mathrm{~mm} \mathrm{NaCl}, 1.5 \mathrm{~mm} \mathrm{MgCl}_{2}, 1 \mathrm{~mm}$ EGTA, $100 \mathrm{~mm} \mathrm{NaF}, 10 \mathrm{~mm} \mathrm{NaPPi}, 10 \%$ glycerol, and $1 \%$ Triton X-100. Protease inhibitors PMSF (1 mM), aprotinin $\left(125000 \mathrm{IU} \mathrm{ml}^{-1}\right)$, and $\mathrm{Na}_{3} \mathrm{VO}_{4}(1 \mathrm{~mm})$ were added to the lysis buffer freshly. Cell lysates were sonicated briefly for three cycles ( $5 \mathrm{~s}$ per cycle, $0^{\circ} \mathrm{C}$ ). Sonicates were incubated on ice for $1 \mathrm{~h}$ and pelleted by centrifugation $(15000 \mathrm{~g} ; 20 \mathrm{~min})$. Protein concentration was determined using Bio-Rad DC Protein Assay Kits. Equal amounts of proteins $(50-100 \mu \mathrm{g})$ were loaded and resolved by $15 \%$ SDS-PAGE and electrotransferred $(110 \mathrm{~V}, 1 \mathrm{~h})$ onto nitrocellulose membranes (Bio-Rad) as previously described (Fraser et al, 2006). Membranes were blocked (at room temperature, $1 \mathrm{~h}$ ) with $5 \%$ Blotto (Tris- $\mathrm{HCl}$ (10 mM; pH 8.0), NaCl (150 mM), Tween 20 $(0.05 \%$, v/v; TBS-Tween 20$)$ containing skim milk (5\%; w/v)), then incubated overnight with primary antibodies iNOS, eNOS, nNOS, and p53 $(1: 1000)$ or GAPDH $(1: 200000))$, and subsequently with the appropriate horseradish peroxidase-conjugated secondary antibody (1:2000 in 5\% Blotto; at room temperature for $1 \mathrm{~h}$ ). The membranes were washed with TBS-Tween 20 thrice ( $15 \mathrm{~min}$ per wash), peroxidase activity was visualised with ECL reagent (Amersham Biosciences), and exposed to Hyperfilm ECL (Amersham Biosciences, Buckinghamshire, UK). Signal intensity was determined densitometrically by Scion Image Software (version 4.02; Scion Corporation, Frederick, MD, USA).

\section{RNA interference}

After plating cells for $18 \mathrm{~h}, 6 \mu \mathrm{l}$ of RiboJuice (Novagen, San Diego, CA, USA) was added to $244 \mu \mathrm{l}$ of RPMI medium without serum. The mixture was vortexed and incubated for $5 \mathrm{~min}$ at room temperature. After incubation, p53 siRNA (Cell Signaling Technology) and control non-targeting siRNA (Dharmacon) at final concentration of $50 \mathrm{~nm}$ were mixed with the transfection reagents. The mixture was incubated at room temperature for $15 \mathrm{~min}$. During this period, the culture media were removed from the cells and the cells were washed once with PBS. The siRNA mixture was added in drop by drop to each well to ensure maximal coverage, and an additional $1250 \mu \mathrm{l}$ of complete media was added to bring the total volume to $1.5 \mathrm{ml}$. The cells were incubated for $6 \mathrm{~h}$ and then the media were removed and replaced with fresh, complete media for $24 \mathrm{~h}$. Downregulation was confirmed by western blot analysis.

\section{Statistical analysis}

Results are expressed as mean \pm s.e.m. of four or five independent experiments. Statistical analysis was performed by one-way or twoway ANOVA using PRISM software (Version 3.0; GraphPad, San Diego, CA, USA). Bartlett's tests were used to establish homogeneity of variance on the basis of differences among s.d. Differences between experimental groups were determined by Bonferroni multiple comparison test. $P<0.05$ was considered to be significant.

\section{RESULTS}

Basal iNOS, eNOS, and nNOS levels are different in CDDP-sensitive (OV2008) cells than in CDDP-resistant $\left(\mathrm{C}^{*} 3^{*}\right)$ cells and are differentially regulated by CDDP in vitro

To assess the protein levels of basal and CDDP-induced NOS in chemosensitive and chemoresistance cells, we cultured OV2008 ovarian cancer cells and the isogenic chemoresistant variant $\mathrm{C} 13^{*}$ in the absence or presence of CDDP $(0-10 \mu \mathrm{M}, 24 \mathrm{~h}$; DMSO 

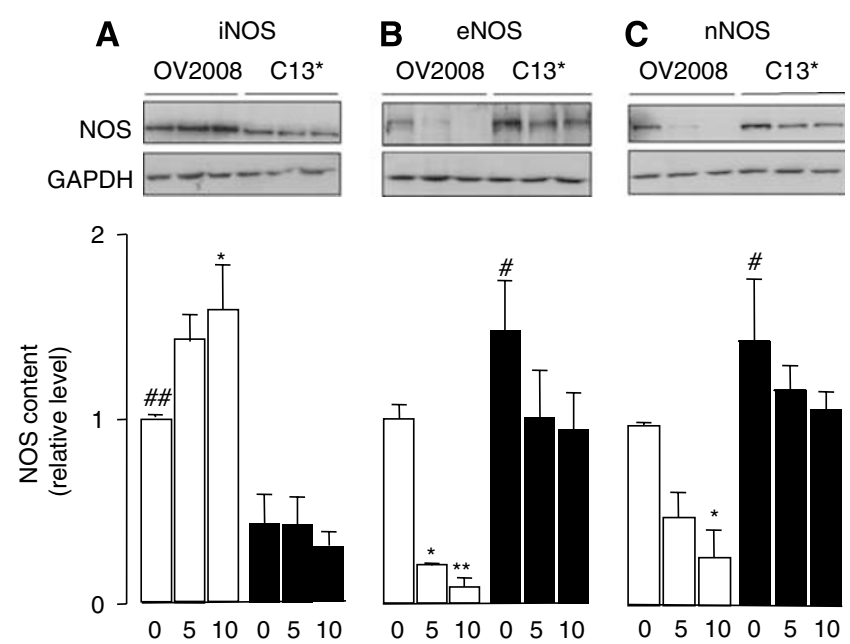

$\operatorname{CDDP}(\mu \mathrm{m})$

Figure I Protein levels of iNOS, eNOS, and nNOS in cisplatin-sensitive OV2008 and in cisplatin-resistant Cl3* cells were determined using western blot analysis. (A) Basal iNOS levels were significantly higher in OV2008 cells compared with Cl3* cells. Cisplatin (CDDP, $10 \mu \mathrm{M}, 24 \mathrm{~h}$ ) significantly upregulated iNOS in OV2008, but not in CI3* cells. (B and C) Basal levels of eNOS and nNOS were significantly lower in OV2008 cells compared with $\mathrm{Cl} 3^{*}$ cells. Cisplatin (5 and $10 \mu \mathrm{M}, 24 \mathrm{~h}$ ) significantly downregulated both eNOS and nNOS in OV2008, but not in C13*, cells. The bar graphs show mean \pm s.e.m. of protein levels of five independent experiments. $* P<0.05$, compared with control (no cisplatin). ${ }^{*} P<0.01$, compared with control (no cisplatin). ${ }^{\#} P<0.05$, comparing basal levels in control OV2008 and in Cl3* cells. ${ }^{\# \#} P<0.01$, comparing basal levels in control OV2008 and in Cl3* cells.

control) and evaluated iNOS, eNOS, and nNOS contents by western blot. As shown in Figure 1A, basal iNOS levels in OV2008 cells are significantly higher than those in $\mathrm{C}^{*} 3^{*}$ cells $(P<0.05)$ and are significantly upregulated by CDDP in the chemosensitive cells, but not in the resistant variant cells $(P<0.01)$. In contrast, basal eNOS and nNOS levels in CDDP-sensitive OV2008 cells were significantly $(P<0.05)$ lower than in CDDP-resistant $\mathrm{C}_{13}{ }^{*}$ cells (Figure $1 \mathrm{~B}$ and $C)$ and were significantly downregulated after CDDP treatment $(P<0.01)$. As observed with iNOS, CDDP failed to significantly alter eNOS and nNOS content in $\mathrm{C}^{*} 3^{*}$ cells, although a slight downwards trend was observed. The data are consistent with the hypothesis that iNOS, eNOS, and nNOS are differentially regulated in chemosensitive (OV2008) and chemoresistant (C13) cells and that CDDP resistance is associated with low iNOS content and high levels of eNOS and nNOS in C13 cells, which are unresponsive to CDDP.

\section{Effects of a NO donor on p53 and CDDP sensitivity}

Because we observed differential regulation of NOS isoforms in chemosensitive and chemoresistant ovarian cancer cells, we next asked whether NO itself could influence sensitivity to CDDPinduced apoptosis. Moreover, as p53 is a key determinant of CDDP sensitivity (Fraser et al, 2003b) and is regulated by the cGMP pathway (Fraser et al, 2006), we also examined the effects of the NO donor SNAP (Bellamy et al, 2002; Fiscus et al, 2002) on basal and CDDP-induced p53 contents. OV2008 or C13* cells were pretreated with SNAP for $24 \mathrm{~h}$, followed by treatment with CDDP for a further $24 \mathrm{~h}$. As shown in Figure 2A, SNAP significantly upregulated p53 content $(P<0.05)$ and induced apoptosis $(P<0.01)$ in OV2008 cells. Interestingly, although CDDP alone also upregulated p53 and induced apoptosis in these cells, cotreatment with SNAP and CDDP resulted in a decrease in p53 content and no further increase in CDDP-induced apoptosis. Similarly, in $\mathrm{C} 13^{*}$ cells, SNAP alone upregulated basal p53 and induced apoptosis in $\mathrm{C}_{13}{ }^{*}$ cells. However, in contrast to the chemosensitive cells, pretreatment with SNAP facilitated the CDDP-induced upregulation of p53 $(P<0.01)$ and sensitised the cells to CDDP-induced apoptosis $(P<0.05)$ (Figure $2 \mathrm{~B})$. These data suggest that high-level NO, released from an NO donor such as SNAP (Bellamy et al, 2002; Fiscus et al, 2002), upregulates p53 and induces apoptosis in resistant ovarian cancer cells and sensitises these cells to pro-apoptotic effects of CDDP.

p53 siRNA reduces NO donor-induced apoptosis in both OV2008 and $\mathrm{C}^{*}{ }^{\star}$ cells and sensitises $\mathrm{C13}{ }^{*}$ cells to CDDP

We and others have demonstrated that p53 is an important determinant of sensitivity to CDDP-induced apoptosis in ovarian cancer cells. To test the role of p53 in the chemosensitising effects of high concentrations of NO, we transfected chemosensitive and chemoresistant cells with p53 siRNA and assessed the effects of p53 downregulation on SNAP-mediated sensitisation to CDDP-induced apoptosis. As shown in Figure 3A, p53 levels were successfully silenced by p53 siRNA, as compared with control siRNA transfection in both OV2008 and $\mathrm{C}^{*} 3^{*}$ cells. Importantly, the downregulation of p53 significantly attenuated SNAP-induced apoptosis in both OV2008 and C13* cells $(P<0.001$ and $P<0.01$, respectively), suggesting that high concentrations of NO induce apoptosis in a p53-dependent manner.

To evaluate whether p53 is implicated in the sensitisation of cells to CDDP-induced apoptosis by high concentrations of NO, we transfected $\mathrm{C}_{13}{ }^{*}$ cells with p53 siRNA (or control) followed by treatment with SNAP and/or CDDP. As shown in Figure 3B and consistent with the results shown in Figure 2B, SNAP sensitised $\mathrm{C}_{13}{ }^{*}$ cells to $\mathrm{CDDP}$-induced apoptosis, and this was significantly suppressed by the downregulation of p53 $(P<0.001)$, suggesting that p53 is necessary for NO donor-induced sensitisation to CDDP.

\section{iNOS is required for CDDP-induced p53 accumulation and apoptosis}

We sought to assess whether iNOS is required for the proapoptotic effects of CDDP. To that end, we treated OV2008 cells with the specific iNOS inhibitor 1400W (Thomsen et al, 1997) $(100 \mu \mathrm{M}, 24 \mathrm{~h})$ and evaluated CDDP-induced p53 content and apoptosis. As shown in Figure 4, 1400W partially blocked CDDPinduced p53 accumulation. However, this effect was observed only with higher concentrations of $1400 \mathrm{~W}$. In contrast, the proapoptotic effects of CDDP were significantly reduced by both higher and lower $(10 \mu \mathrm{M})$ concentrations of $1400 \mathrm{~W}$, suggesting that CDDP-induced apoptosis is dependent on endogenous iNOS activity.

Inhibition of all endogenous NOSs does not alter CDDP-induced p53 content in CDDP-resistant $\mathrm{C} 13^{*}$ cells, but sensitises them to CDDP

Because CDDP-resistant $\mathrm{C} 13^{*}$ cells show higher basal protein levels of eNOS and nNOS and are resistant to CDDP-induced downregulation of eNOS and nNOS, we hypothesised that eNOS/nNOS activity may play an important role in the resistance to CDDP in $\mathrm{C} 13^{*}$ cells. To test this hypothesis, L-NAA, a general NOS inhibitor that inhibits all three isoforms of NOS, was used (Reif and McCreedy, 1995). Unlike 1400W, which is highly specific for iNOS, none of the commercially available inhibitors of eNOS or nNOS are highly specific. Thus, to effectively inhibit eNOS and nNOS in CDDP-resistant $\mathrm{C} 13^{*}$ cells, we used the multitargeted NOS inhibitor L-NAA.

Figure 5 shows western blot analysis of $\mathrm{C} 3^{*}$ cells pretreated with L-NAA $(0,10$, or $100 \mu \mathrm{M})$ for $24 \mathrm{~h}$, followed by $\operatorname{CDDP}(0,5$, or 
A
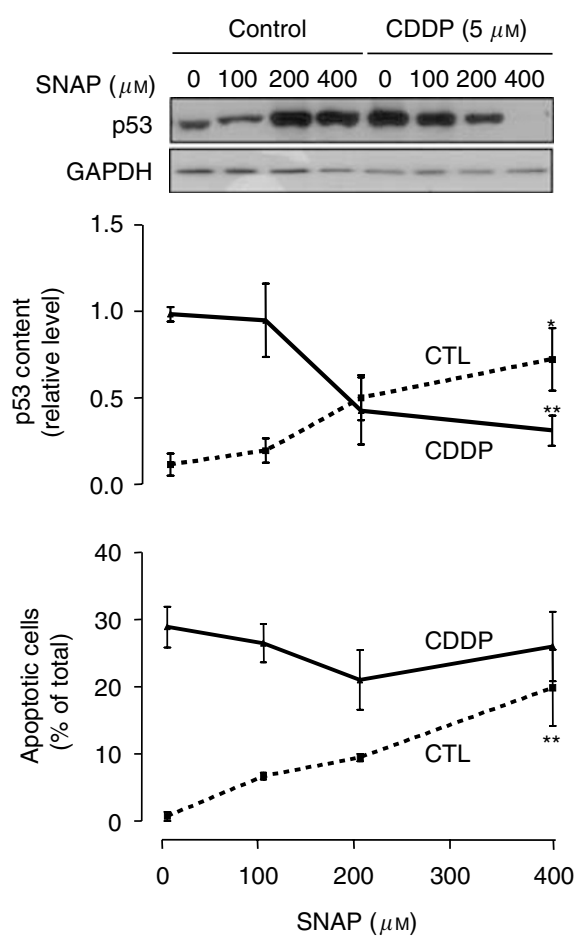

B

C13* cells
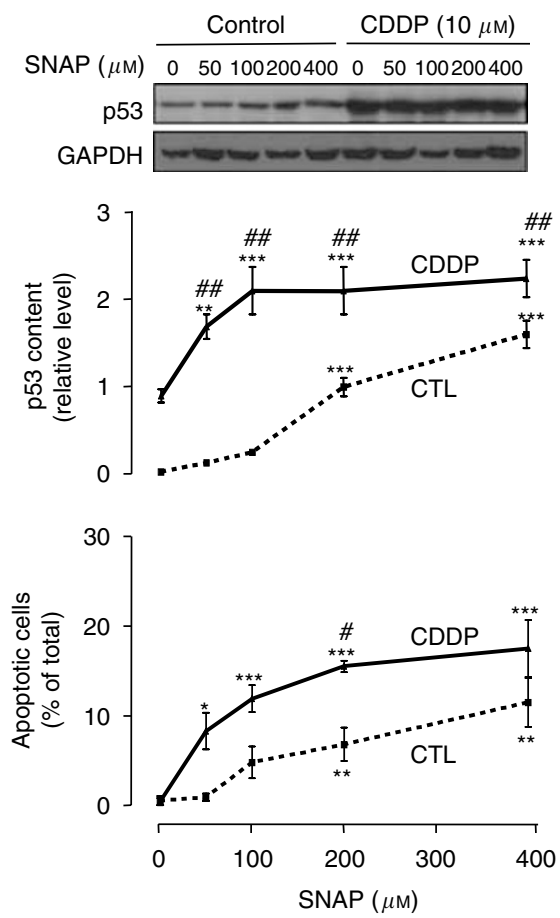

Figure 2 (A) OV2008 cells were cultured with SNAP (0, 100, 200, $400 \mu \mathrm{M})$ for $24 \mathrm{~h}$, followed by $5 \mu \mathrm{M}$ cisplatin (CDDP) for another $24 \mathrm{~h}$. p53 protein content was assessed by western blot analysis. Results were normalised for protein loading by re-probing with anti-GAPDH antibody. Western blots are representative of four independent experiments. Graphs show mean \pm s.e.m. of protein contents of four independent experiments. S-nitroso- $N$ acetylpenicillamine alone $(400 \mu \mathrm{M}$, $* P<0.05)$ increased basal p53 content, but decreased cisplatin-induced upregulation of p53 (** $\mathrm{P}<0.0 \mathrm{l})$ in chemosensitive OV2008 cells. S-nitroso-N-acetylpenicillamine $(400 \mu \mathrm{M})$ alone significantly $(* * P<0.0 \mathrm{I})$ increased apoptosis. However, when used in combination with cisplatin, SNAP did not further enhance cisplatin-induced apoptosis. (B) CI3* cells were cultured with SNAP $(0,50,100,200,400 \mu \mathrm{M})$ for $24 \mathrm{~h}$, followed by $10 \mu \mathrm{M}$ cisplatin for another $24 \mathrm{~h}$. Westem blots are representative of five independent experiments. Graphs show mean \pm s.e.m. of p53 protein content and apoptosis of five independent experiments. S-nitroso-N-acetylpenicillamine $(200$ and $400 \mu \mathrm{M})$ alone significantly $(* * * *<<00$ I) upregulated p53 levels. With cisplatin co-treatment, SNAP at 50, 100, 200, and $400 \mu \mathrm{M}$ significantly (**P $<0.0 \mathrm{I}$, **** $<<0.00 \mathrm{I}$ ) increased p53 levels above control. p53 upregulation induced by SNAP plus cisplatin was significantly $\left({ }^{\#} P<0.01\right.$ I) larger than with SNAP alone. S-nitroso-N-acetylpenicillamine $(200$ and $400 \mu \mathrm{M})$ alone significantly (**P $\left.<0.0 \mathrm{I}\right)$ induced apoptosis and sensitised chemoresistant CI3* cells to cisplatin-induced apoptosis (SNAP at $200 \mu \mathrm{M},{ }^{\#} P<0.05$ ).

A
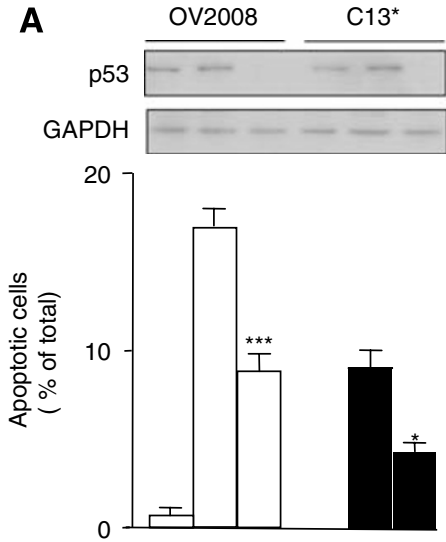

p53 siRNA (50 nm)

SNAP $(400 \mu \mathrm{m})$
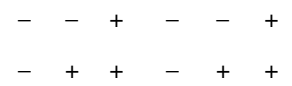

B
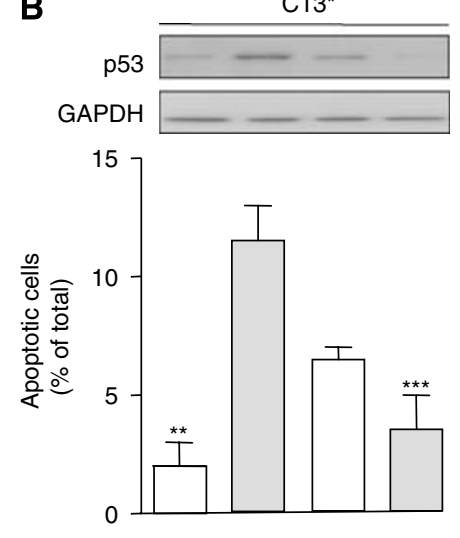

p53 siRNA (50 nm)

SNAP $(200 \mu \mathrm{m})$

Figure 3 (A) Both OV2008 and Cl3* cells were transfected with p53-specific siRNA (50 nM; scrambled sequence as control) for 24h, and subsequently treated with $400 \mu \mathrm{M}$ SNAP for another $24 \mathrm{~h}$. Western blots are representative of four independent experiments. Graphs show mean \pm s.e.m. of four independent experiments. Western blots show that p53 was successfully knocked down. $* P<0.05$, ***P $<0.001$, compared with SNAP treatment alone. (B) CI3* cells were transfected with p53-specific siRNA ( $50 \mathrm{nM}$; scrambled sequence as control) for $24 \mathrm{~h}$, and subsequently treated with $200 \mu \mathrm{M}$ SNAP before cisplatin (CDDP, I0 $\mu \mathrm{M}$ ) treatments for another $24 \mathrm{~h}$. Western blots are representative of four independent experiments and graphs show mean \pm s.e.m. from four independent experiments. **P $<0.01$, compared with SNAP alone, ***P $<0.001$, compared with SNAP plus cisplatin (without siRNA). 

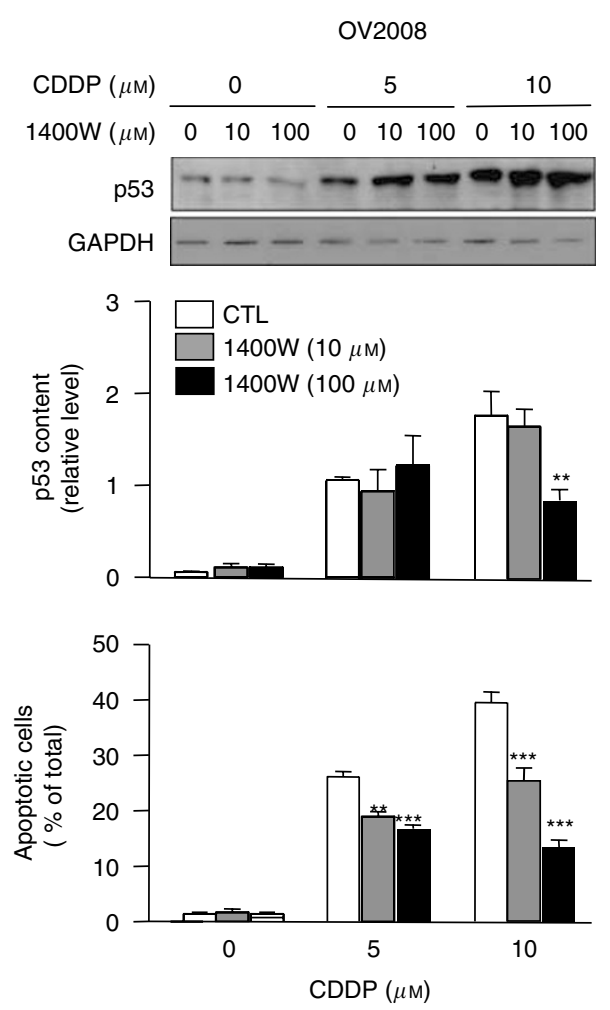

Figure 4 OV2008 cells were cultured with I400W (0, 10, 100 $\mu \mathrm{M})$ for $24 \mathrm{~h}$ and further treated with cisplatin (CDDP; 0, 5, $10 \mu \mathrm{M})$ for another $24 \mathrm{~h}$. Protein content of p53 was assessed by western blot analysis and apoptosis by Hoechst staining. Western blots are representative of four independent experiments. Graphs show mean \pm s.e.m. of four independent experiments. $* * P<0.01$, *** $P<0.001$, compared with control.

$10 \mu \mathrm{M}$ ) for a further $24 \mathrm{~h}$. There was no significant effect of L-NAA on p53 levels in any of the CDDP-treated groups, suggesting that neither eNOS nor nNOS affect p53 content. In contrast, L-NAA (both 10 and $100 \mu \mathrm{M}$ ) dramatically enhanced the pro-apoptotic effects of CDDP. These data suggest that low-level NO generated by eNOS and nNOS contributes to the suppression of CDDP-induced apoptosis in $\mathrm{C}_{13}{ }^{*}$ cells.

\section{DISCUSSION}

The present study demonstrates that protein levels of the different NOS isoforms or co-treatment with the NO donor SNAP regulates induction of apoptosis and sensitisation to CDDP in human ovarian cancer cells. CDDP upregulates iNOS protein levels and markedly downregulates eNOS and nNOS protein levels in CDDPsensitive OV2008 cells but has no significant effect on the levels of the three NOSs in CDDP-resistant $\mathrm{C}^{*} 3^{*}$ cells, suggesting that differential regulation of the NOSs may contribute to CDDP resistance. The data using NOS inhibitors suggest that iNOS contributes to CDDP-induced apoptosis in OV2008 cells, whereas eNOS/nNOS contributes to CDDP-resistant in $\mathrm{C} 13^{*}$ cells.

Endogenous NO is produced in mammalian cells by the three NOSs, namely eNOS (NOS3), originally found constitutively expressed in endothelial cells; iNOS (NOS2), first found to be induced by cytokines in activated macrophages; and nNOS (NOS1), originally discovered to be constitutively expressed in neural cells (Forstermann et al, 1995; Fiscus, 2002; Fiscus et al, 2002). All of these NOS isozymes have now been found in many other cell types. Typically, eNOS and nNOS produce NO at low or physiological levels, which play important roles in the normal
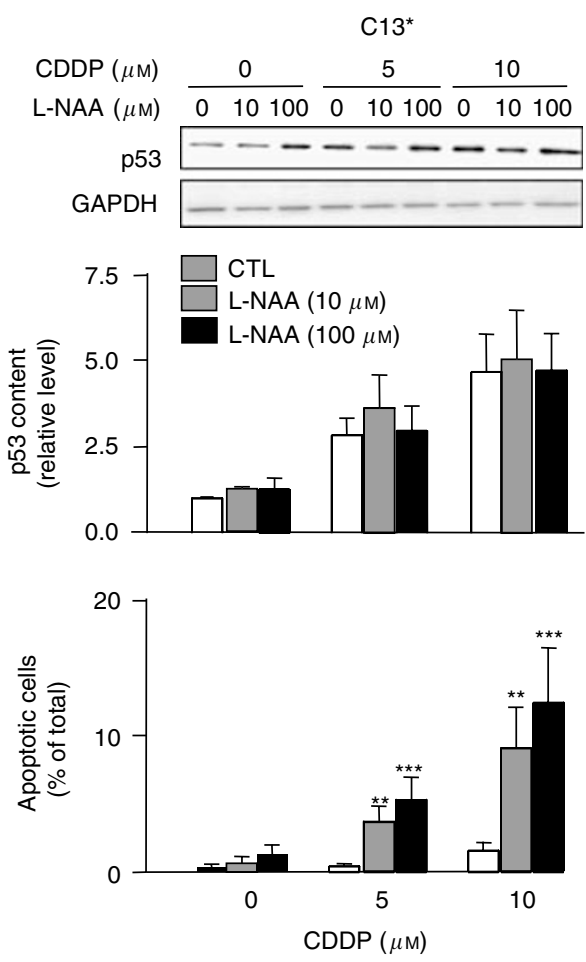

Figure 5 Inhibition of all NOSs using $N^{G}$-amino-L-arginine (L-NAA; 0, I0, $100 \mu \mathrm{M})$ for $24 \mathrm{~h}$, followed by cisplatin $(0,5,10 \mu \mathrm{M})$ for additional $24 \mathrm{~h}$. Western blots are representative of four independent experiments and graphs shows mean \pm s.e.m. from four independent experiments. *** $P<0.01$, **** $P<0.001$

regulation of the cardiovascular and neural systems (Fiscus, 2002; Fiscus et al, 2002). Low-level NO (e.g., 1-20 nM) binds to the heme moiety of sGC, increasing its enzymatic activity, which in turn results in the elevation of cGMP levels and increased activity of PKG in mammalian cells. Stimulation of the sGC/cGMP/PKG pathway by low-level NO contributes to cytoprotective/antiapoptotic effects in many types of mammalian cells (Fiscus, 2002; Fiscus et al, 2002). In contrast, iNOS typically produces toxicity-associated NO. The toxicity may result from NO combining with superoxide anion, forming peroxynitrite, a powerful oxidising substance that induces oxidative and nitrosative stress, and apoptosis in mammalian cells (Fiscus, 2002; Fiscus et al, 2002). The present study shows that all three isoforms of NOS are expressed in human ovarian cancer cells and that the NOSs are differentially expressed in CDDP-sensitive and CDDP-resistant cells, suggesting potential involvement in chemoresistance.

Previous reports have demonstrated that pro-inflammatory cytokines induced iNOS expression and apoptosis in ovarian cancer cells (Son and Hall, 2000; Rieder et al, 2001). The current study extends these findings by demonstrating that the proapoptotic effects of iNOS may be mediated, in part, through a p53dependent pathway. This finding may have significant implications with regard to the molecular mechanisms of chemosensitivity in ovarian cancer cells, as p53 plays a key role in this process (Fraser et al, 2003b). This hypothesis is further supported by the observations that in normal human fibroblasts and liver epithelial cells, iNOS induction stimulates p53 accumulation and apoptosis (Forrester et al, 1996). Many tumour cells express higher levels of iNOS and it is believed that NO generated by iNOS contributes to p53 upregulation and induction of apoptosis in cancer cells (Ambs et al, 1997). However, to date, reports showing direct functional evidence linking NO, p53, and chemosensitivity in ovarian cancer 
cells have been lacking. Thus, the present study represents the first report directly demonstrating the role of p53 in high-level NOinduced apoptosis (using siRNA gene knockdown) and the role of the three endogenous NOSs in regulating sensitivity to CDDP in ovarian cancer cells.

The present study shows that the NO donor SNAP, when used at higher concentrations (200 and $400 \mu \mathrm{M})$ that generate toxic levels of NO, induces apoptosis in both CDDP-sensitive (OV2008) and CDDP-resistant $\left({\left.\mathrm{C} 13^{*}\right)}^{*}\right.$ ovarian cancer cells. Furthermore, SNAP $(50-400 \mu \mathrm{M})$ caused concentration-dependent enhancement of CDDP-induced p53 upregulation in $\mathrm{C}^{*} 3^{*}$ cells, sensitising these cells to pro-apoptotic effects of CDDP. Gene knockdown with p53 siRNA prevented this sensitisation, demonstrating that high concentrations of NO contribute to CDDP sensitivity through a p53-dependent mechanism.

Although depletion of endogenous NO in melanoma cells by NO scavengers enhanced CDDP-induced apoptosis and cell cycle arrest in a p53-dependent manner (Tang and Grimm, 2004), a direct connection between p53 and CDDP-induced apoptosis was not established. The present study shows that CDDP sensitisation induced by high levels of NO donor in ovarian cancer cells directly involves p53 upregulation, illustrated by our p53 siRNA gene knockdown experiments (Figure 3 ). In contrast, CDDP sensitisation induced by inhibition of all three forms of NOS in $\mathrm{C}_{13}{ }^{*}$ ovarian cancer cells (Figure 5) did not change p53 levels in ovarian cancer cells. Thus, the role of p53 in CDDP sensitisation appears to be different in melanoma and ovarian cancer cells.

Our data suggest that endogenous eNOS/nNOS activity in ovarian cancer cells, especially CDDP-resistant cells, produces lowlevel NO that protects them against induction of apoptosis, thus contributing to resistance against CDDP. Although iNOS could potentially also produce low-level NO favouring cytoprotection, the role of iNOS in ovarian cancer cells appears to be different from that of eNOS/nNOS; that is, iNOS contributes to, rather than suppresses, CDDP-induced apoptosis. This difference may relate to different localisation of the NOSs or different products generated by the NOSs (e.g., eNOS/nNOS generating cytoprotective NO, whereas iNOS generating cytotoxic forms of NO, such as peroxynitrite). Further studies will be needed to fully elucidate the different roles of the three NOSs in chemosensitive and chemoresistant ovarian cancer cells.

Overall, our data suggest a novel role for different NOSs in the regulation of apoptosis and sensitivity to CDDP in human ovarian cancer cells. The three NOS isoforms are regulated differentially by CDDP in CDDP-resistant and CDDP-sensitive ovarian cancer cells, which may contribute to chemoresistance. Inhibition of all NOSs in CDDP-resistant cells dramatically sensitises these cells to CDDP-induced apoptosis. The data suggest potential new ways of sensitising chemoresistant ovarian cancer cells to the therapeutic effects of CDDP.

\section{ACKNOWLEDGEMENTS}

This work was supported by grants awarded to BKT by the Canadian Institutes of Health Research (CIHR, MOP-15691), to RRF by the Research Grants Council of Hong Kong (Grant CUHK4169/02M), and to ELL by the Overseas Academic Activities Committee from The Chinese University of Hong Kong. MF was the recipient of a Canada Graduate Scholarship Doctoral Research Award from CIHR.

\section{REFERENCES}

Ambs S, Hussain SP, Harris CC (1997) Interactive effects of nitric oxide and the $\mathrm{p} 53$ tumor suppressor gene in carcinogenesis and tumor progression. FASEB J 11: $443-448$

Bellamy TC, Griffiths C, Garthwaite J (2002) Differential sensitivity of guanylyl cyclase and mitochondrial respiration to nitric oxide measured using clamped concentrations. J Biol Chem 277: 31801-31807

Chan SL, Fiscus RR (2003) Guanylyl cyclase inhibitors NS2028 and ODQ and protein kinase G (PKG) inhibitor KT5823 trigger apoptotic DNA fragmentation in immortalized uterine epithelial cells: anti-apoptotic effects of basal cGMP/PKG. Mol Hum Reprod 9: $775-783$

Cheng Chew SB, Leung PY, Fiscus RR (2003) Preincubation with atrial natriuretic peptide protects NG108-15 cells against the toxic/proapoptotic effects of the nitric oxide donor $S$-nitroso- $N$-acetylpenicillamine. Histochem Cell Biol 120: 163-171

Fiscus RR (2002) Involvement of cyclic GMP and protein kinase G in the regulation of apoptosis and survival in neural cells. Neurosignals 11: $175-190$

Fiscus RR, Tu AW, Chew SB (2001) Natriuretic peptides inhibit apoptosis and prolong the survival of serum-deprived PC12 cells. Neuroreport 12: $185-189$

Fiscus RR, Yuen JP, Chan SL, Kwong JH, Chew SB (2002) Nitric oxide and cyclic GMP as pro- and anti-apoptotic agents. J Card Surg 17: $336-339$

Forrester K, Ambs S, Lupold SE, Kapust RB, Spillare EA, Weinberg WC, Felley-Bosco E, Wang XW, Geller DA, Tzeng E, Billiar TR, Harris CC (1996) Nitric oxide-induced p53 accumulation and regulation of inducible nitric oxide synthase expression by wild-type p53. Proc Natl Acad Sci USA 93: 2442-2447

Forstermann U, Gath I, Schwarz P, Closs EI, Kleinert H (1995) Isoforms of nitric oxide synthase. Properties, cellular distribution and expressional control. Biochem Pharmacol 50: $1321-1332$

Fraser M, Bai T, Tsang BK (2008) Akt promotes cisplatin resistance in human ovarian cancer cells through inhibition of p53 phosphorylation and nuclear function. Int J Cancer 122: 534-546
Fraser M, Chan SL, Chan SS, Fiscus RR, Tsang BK (2006) Regulation of p53 and suppression of apoptosis by the soluble guanylyl cyclase/cGMP pathway in human ovarian cancer cells. Oncogene 25: 2203-2212

Fraser M, Leung B, Jahani-Asl A, Yan X, Thompson WE, Tsang BK (2003a) Chemoresistance in human ovarian cancer: the role of apoptotic regulators. Reprod Biol Endocrinol 1: 66

Fraser M, Leung BM, Yan X, Dan HC, Cheng JQ, Tsang BK (2003b) p53 is a determinant of X-linked inhibitor of apoptosis protein/Akt-mediated chemoresistance in human ovarian cancer cells. Cancer Res 63: $7081-7088$

Mannick JB, Asano K, Izumi K, Kieff E, Stamler JS (1994) Nitric oxide produced by human B lymphocytes inhibits apoptosis and Epstein-Barr virus reactivation. Cell 79: 1137-1146

Mannick JB, Miao XQ, Stamler JS (1997) Nitric oxide inhibits Fas-induced apoptosis. J Biol Chem 272: $24125-24128$

Reif DW, McCreedy SA (1995) $\mathrm{N}$-nitro-L-arginine and $\mathrm{N}$-monomethyl-Larginine exhibit a different pattern of inactivation toward the three nitric oxide synthases. Arch Biochem Biophys 320: 170-176

Rieder J, Jahnke R, Schloesser M, Seibel M, Czechowski M, Marth C, Hoffmann G (2001) Nitric oxide-dependent apoptosis in ovarian carcinoma cell lines. Gynecol Oncol 82: 172 - 176

Sasaki H, Sheng Y, Kotsuji F, Tsang BK (2000) Down-regulation of X-linked inhibitor of apoptosis protein induces apoptosis in chemoresistant human ovarian cancer cells. Cancer Res 60: 5659-5666

Siddik ZH (2003) Cisplatin: mode of cytotoxic action and molecular basis of resistance. Oncogene 22: 7265-7279

Son KK, Hall KJ (2000) Nitric oxide-mediated tumor cell killing of cisplatin-based interferon-gamma gene therapy in murine ovarian carcinoma. Cancer Gene Ther 7: 1324-1328

Tang CH, Grimm EA (2004) Depletion of endogenous nitric oxide enhances cisplatin-induced apoptosis in a p53-dependent manner in melanoma cell lines. J Biol Chem 279: 288-298

Tejedo JR, Ramirez R, Cahuana GM, Rincon P, Sobrino F, Bedoya FJ (2001) Evidence for involvement of c-Src in the anti-apoptotic action of nitric oxide in serum-deprived RINm5F cells. Cell Signal 13: 809-817 
Thomsen LL, Miles DW (1998) Role of nitric oxide in tumour progression: lessons from human tumours. Cancer Metastasis Rev 17: 107-118

Thomsen LL, Scott JM, Topley P, Knowles RG, Keerie AJ, Frend AJ (1997) Selective inhibition of inducible nitric oxide synthase inhibits tumor growth in vivo: studies with $1400 \mathrm{~W}$, a novel inhibitor. Cancer Res 57: 3300-3304

Wink DA, Hanbauer I, Krishna MC, DeGraff W, Gamson J, Mitchell JB (1993) Nitric oxide protects against cellular damage and cytotoxicity from reactive oxygen species. Proc Natl Acad Sci USA 90: 9813-9817
Wink DA, Vodovotz Y, Cook JA, Krishna MC, Kim S, Coffin D, DeGraff W, Deluca AM, Liebmann J, Mitchell JB (1998) The role of nitric oxide chemistry in cancer treatment. Biochemistry (Mosc) 63: $802-809$

Yuan ZQ, Feldman RI, Sussman GE, Coppola D, Nicosia SV, Cheng JQ (2003) AKT2 inhibition of cisplatin-induced JNK/p38 and Bax activation by phosphorylation of ASK1: implication of AKT2 in chemoresistance. J Biol Chem 278: 23432 - 23440 